\title{
IDENTIFICAÇÃO DE PROCESSOS EM TEMPO REAL VIA MODELO ARMAX
}

\author{
C. B. CAVALCANTI ${ }^{1}$, J. N. SILVA ${ }^{1}$ \\ ${ }^{1}$ Universidade Federal de Campina Grande, Departamento de Engenharia Química \\ E-mail para contato: nilton.silva@ufcg.edu.br
}

\begin{abstract}
RESUMO - Como consequência à grande demanda das indústrias por controladores, o algoritmo de controle PID (Proporcional, Integral e Derivativo), por ser de fácil entendimento, é o mais difundido em todo o mundo. No entanto, estimativas mostram que apenas $20 \%$ das malhas de controle industriais funcionam adequadamente na tentativa de minimizar os efeitos da variabilidade do processo. No intuito de contribuir para um melhor desempenho dessa classe de controladores, este trabalho busca inserir a metodologia de identificação de ARMAX ("AutoRegressive Moving Average with eXogeneus variable"), em tempo real, para geração do modelo recursivo do processo, o qual será utilizado na técnica de autossintonia de controladores PID. A identificação foi realizada utilizando o método dos mínimos quadrados estendido com predição de erros, aplicado a um sistema de separação de metanol e água, através de um vaso Flash. As malhas de controle de nível, temperatura e pressão também compõem o sistema. Tal procedimento foi implementado em plataforma Visual Basic for Applications (VBA) utilizando a comunicação Object Linking and Embedding (OLE) com o Aspen Dynamics ${ }^{\circledR}$. A ferramenta desenvolvida mostra desempenho satisfatório quanto a atingir melhor ajuste em relação à diferença entre o modelo e processo.
\end{abstract}

\section{INTRODUÇÃO}

O primeiro controlador PID data de 1890, baseado a partir de um projeto do governo americano para guiar automaticamente um navio. Na sequência, foram criados vários modelos de navios com esta tecnologia. Segundo Ogata (2003) a primeira publicação contendo análise teórica de um controlador PID foi feita por Nicolas Minorsky em 1922.

O controle PID é ainda hoje predominante no meio industrial. Mais que $90 \%$ de todas as malhas existentes são do tipo PI/PID atingindo uma larga faixa de aplicações: controle de processos, drivers para motores, indústria automobilística, controladores de voo, pilotos automáticos, instrumentação, entre outros ( ̊ström e Hägglund, 2001). Ainda que seja muito usado, este não funciona de forma totalmente satisfatória em algumas aplicações industriais. As principais causas desse desempenho insatisfatório, de acordo com Silva (2013), correspondem a definição incorreta dos parâmetros de sintonia, como também a escolha de emparelhamentos variáveis de processo (Process Variable - PV), manipulada (Manipulated Variable- MV) inadequados em termos de estabilidade. 
Essa realidade mostra que é importante o desenvolvimento de ferramentas que melhorem o desempenho das malhas de controle. Neste contexto, o presente estudo considera a programação, em plataforma VBA, do modelo de identificação via ARMAX ("AutoRegressive Moving Average with eXogeneus variable"), com a estimação on-line de seus parâmetros, utilizando o método dos mínimos quadrados recursivo (RLSM), com atuação na plataforma Aspen Dynamic ${ }^{\circledR}$.

\section{FUNDAMENTAÇÃO TEÓRICA}

\subsection{Controle Proporcional, Integral e Derivativo}

De acordo com Åström e Hägglund, (1995) o controlador PID é, de longe, o mais comumente utilizado, sendo responsável pela resolução de, em média, 90 a 95\% de todos os problemas deste setor. Esse combina em sua estrutura a ação de controle proporcional, integral e derivativo.

A ação de controle proporcional é simplesmente uma resposta proporcional ao erro de controle. Esta ação, quando utilizada individualmente pode gerar um erro estacionário (offset) (Campos e Teixeira, 2010).

Por causa da ação integral, o controlador consegue fazer a saída do processo acompanhar o sinal de referências, isto é, minimizar o desvio do processo em relação ao setpoint. Uma desvantagem desta ação é o fato de que se um erro do mesmo persistir, o termo integral será grande e, eventualmente, a saída do controlador saturará. (reset windup). Nessa situação, uma limitação física (válvula de controle totalmente aberta ou totalmente fechada) não o deixará reduzir o erro a zero.

Já a ação de controle derivativa compensa o resultado saturador da ação integral, pois essa tende a aumentar a estabilidade relativa do sistema e simultaneamente torna a resposta do sistema mais rápida, devido ao seu efeito de antecipação. A desvantagem desta ação é o fato de amplificar os sinais de ruído, o que pode causar uma saturação dos atuadores do sistema.

\subsection{Modelo Recursivo do Processo}

Ogata (2003) definiu o modelo matemático de sistemas dinâmicos como sendo o conjunto de equações que representam a dinâmica de um sistema precisamente bem. Este é o primeiro passo para a representação de sistemas dinâmicos, sendo considerada a parte mais importante para a identificação.

Existem diversos modelos que podem representar um sistema de maneiras diferentes, a escolha vai depender da perspectiva a ser considerada. Alguns dos utilizados para sistemas lineares são funções transferência, modelos de variáveis de estado e os autoregressivos, estes com entradas exógenas (ARX) ou com média móvel e entradas exógenas (ARMAX), (Silva, 2013).

A identificação via ARMAX é uma ferramenta padrão em estudos de teorias de controle e em econometria tanto para descrição de sistemas como para projeto de controladores. 


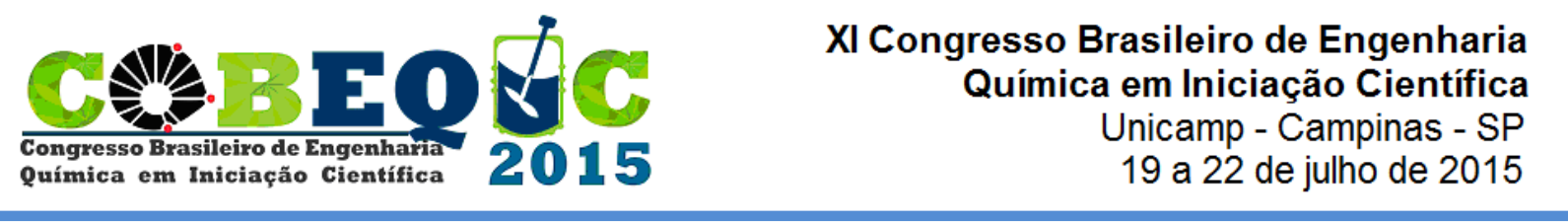

\section{METODOLOGIA}

\subsection{Materiais}

Visto que o trabalho a ser desenvolvido é de natureza teórica e prática, os materiais incluídos estão diretamente envolvidos, estes são ferramentas computacionais na modelagem e simulação do processo. Os softwares utilizados foram o Aspen Dynamics ${ }^{\circledR}$ e o VBA. O sistema de estudo corresponde à separação metanol e água, através de um vaso Flash, com malhas de controle de temperatura, pressão e nível.

\subsection{Métodos}

O sistema estudado, composto por um vaso Flash, uma bomba, um trocador de calor e seis válvulas, o fluxograma encontra-se na Figura 1 a seguir.

Figura 1 - Fluxograma do sistema avaliado no Aspen Plus ${ }^{\circledR}$.

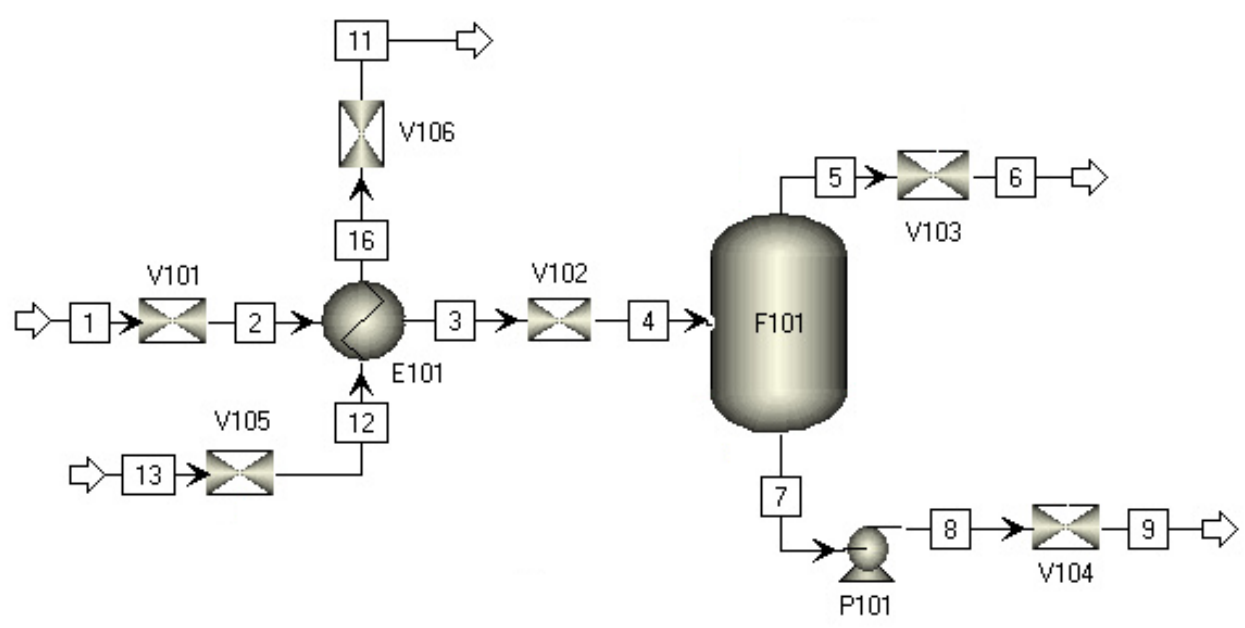

Primeiramente foi feita uma simulação no Aspen Plus ${ }^{\circledR}$ (estado estacionário), fazendo as devidas especificações em cada bloco e correntes de entrada. Uma mistura de metanol e água a $310 \mathrm{~K}, 1,2159$ bar e a uma vazão de $41,3005 \mathrm{kmol} / \mathrm{h}$ entram no sistema, a uma fração molar de 0,46 de metanol. Já a corrente de alimentação quente do trocador de calor contém apenas vapor de água a $510 \mathrm{~K}, 2$ bar e $40 \mathrm{kmol} / \mathrm{h}$. No trocador de calor e no vaso Flash a temperatura das correntes frias foram especificadas, ambas iguais a $342 \mathrm{~K}$. Para o trocador de calor a queda de pressão da corrente quente foi igual a 1,5 bar, nele encontram-se as fases líquida e gás e o mesmo não perde calor para o meio. Em seguida a geometria do vaso Flash é especificada, com comprimento igual a $3 \mathrm{~m}$ e diâmetro $2,5 \mathrm{~m}$, sendo este vertical do tipo Flat.

Ao fim das especificações, o sistema foi exportado para o Aspen Dynamics ${ }^{\circledR}$, onde foram adicionados os controladores PID de nível, temperatura e pressão, de modo que os setpoints estabelecidos foram de $2,125 \mathrm{~m}, 68,85^{\circ} \mathrm{C}$ e 0,8196 bar, respectivamente. Em seguida a conexão com $V B A$ foi estabelecida baseada no algoritmo simplificado mostrado na Figura 2. 
Figura 2 - Algoritmo simplificado do programa desenvolvido no VBA.
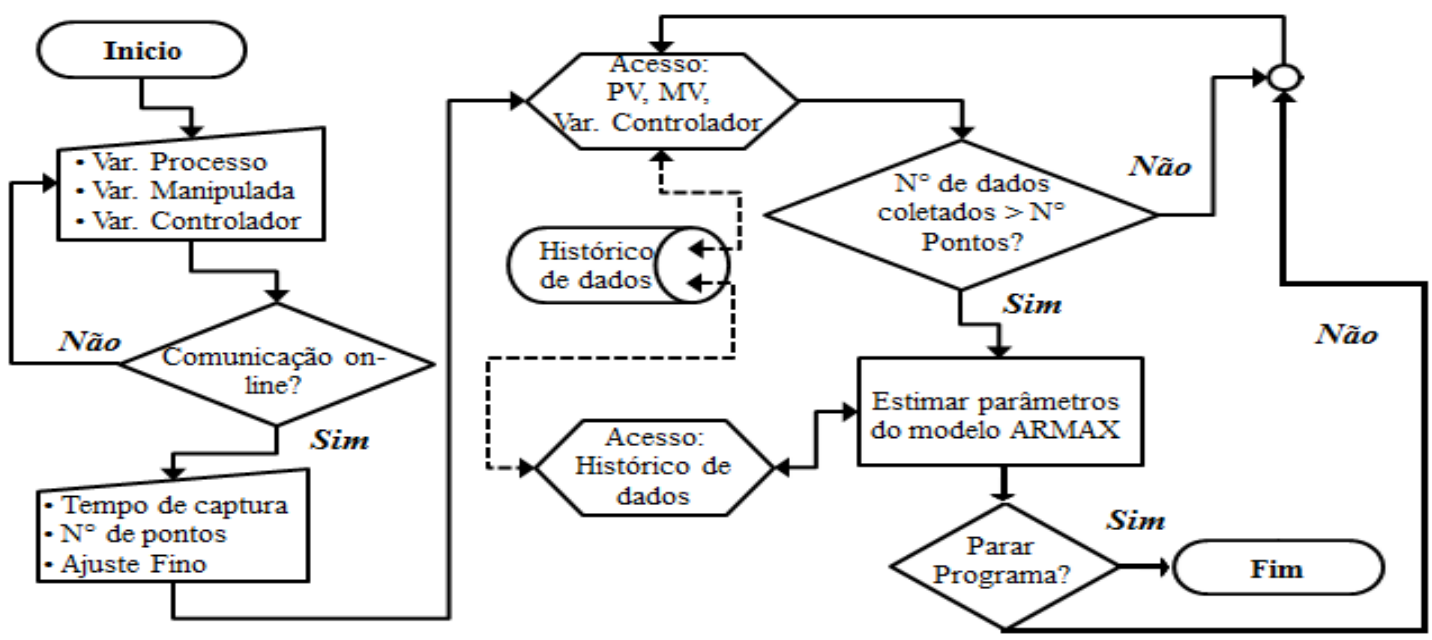

Inicialmente ocorre a entrada manual das variáveis de processo (PV), manipulada (MV) e do controlador (ranges das PV e MV) a serem analisadas para a malha de controle. No passo seguinte, verifica-se a conexão para a aquisição dos dados das variáveis através da comunicação OLE, a partir dos dados da planta simulada no Aspen Dynamics ${ }^{\circledR}$. A tecnologia de comunicação Object Linking and Embedding (OLE) é suportada por ambas as plataformas. $\mathrm{O}$ acesso às variáveis internas na simulação foi possível considerando a linha de código padrão como mostrado na Tabela 1 a seguir.

Tabela 1 - Código de comunicação entre VBA e Aspen Dynamics ${ }^{\circledR}$.

Acesso Input Output ='Aspen Dynamics Language'|'path *.dynf'!'\Object Variable.Value'

A partir da definição dos parâmetros de tempo de captura, número de pontos e ajuste fino desejados, inicia-se o acesso às variáveis $\mathrm{PV}, \mathrm{MV}$ e de controle, sendo continuamente armazenado como histórico de dados. Quando a quantidade de pontos coletados for superior à quantidade de pontos determinados $(n)$, com acesso ao histórico de dados, será iniciado o processamento de estimação dos parâmetros pelo modelo de identificação $A R M A X$ com uso do método dos mínimos quadrados recursivos, que se trata do cálculo do um parâmetro futuro baseado nas informações obtidas no tempo anterior, isso se dá através do operador shift-back (operador atraso), desta forma criando uma memória da variável, replicando em um histórico de dados.

\section{RESULTADOS E DISCUSSÕES}

Após feita a simulação no Aspen Plus ${ }^{\circledR}$, a mesma foi exportada para o Aspen Dynamics ${ }^{\circledR}$, nela o sistema opera em estado transiente, isto é, representa como o processo se comportaria em um teste real, mostrando sua dinâmica, como mostra a Figura 3. Neste, foram implementados os controladores de temperatura, pressão e nível, como já esclarecido anteriormente. 
Figura 3 - Modelo dinâmico da planta separação de metanol e água.

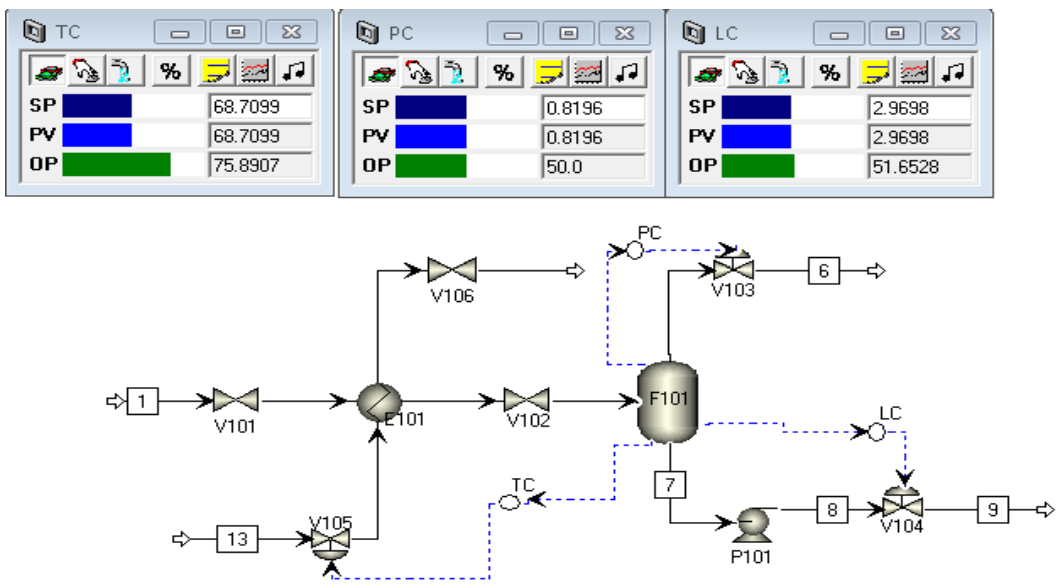

Na plataforma $V B A$ foi desenvolvida a ferramenta de comunicação com o Aspen Dynamics ${ }^{\circledR}$, em que o $V B A$ produz um modelo do processo, através da identificação $A R M A X$, a partir de dados coletados em um tempo $k$-i, sendo $i=1, \ldots, n$, onde o mesmo estima o valor para o tempo $k$.

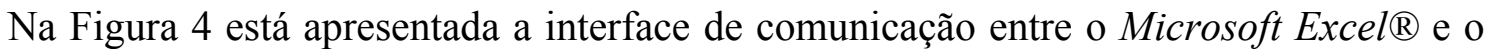
Aspen Dynamics ${ }^{\circledR}$. A configuração das variáveis de comunicação inicia-se com definição do endereço onde se encontra o arquivo da simulação do processo analisado. Após a inserção dessas variáveis, o botão "Start Communication" permite verificar a comunicação com o Aspen Dynamics ${ }^{\circledR}$ com acesso aos dados do sistema. A partir disso, uma vez especificada adequadamente os nomes objetos das variáveis, são obtidos os valores das variáveis. Ao longo do funcionamento da ferramenta proposta, os valores para as variáveis controladas e manipuladas são atualizados.

Figura 4 - Interface de comunicação da ferramenta desenvolvida no Microsoft Excel®.

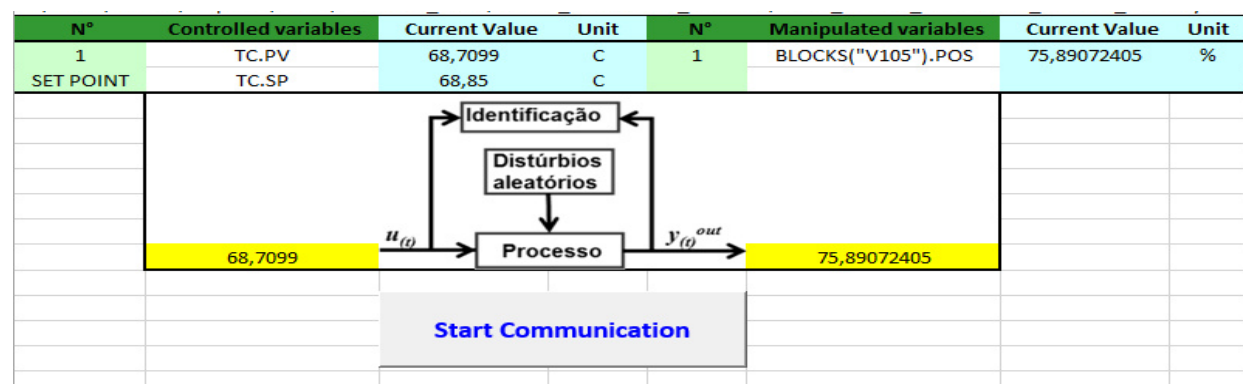

Na Figura 5 está representada a interface de análise do comportamento dinâmico da PV simulada e estimada pelo modelo. A conexão da ferramenta em $V B A$ com a simulação no Aspen Dynamics ${ }^{\circledR}$ realiza-se com acionamento do botão Iniciar, iniciando o algoritmo apresentado na Figura 2, com tempo de captura de $0,1 \mathrm{~s}$ e um total de 30 pontos. A partir desse evento são capturadas as informações necessárias para as etapas de estimação dos parâmetros do modelo ARMAX. 


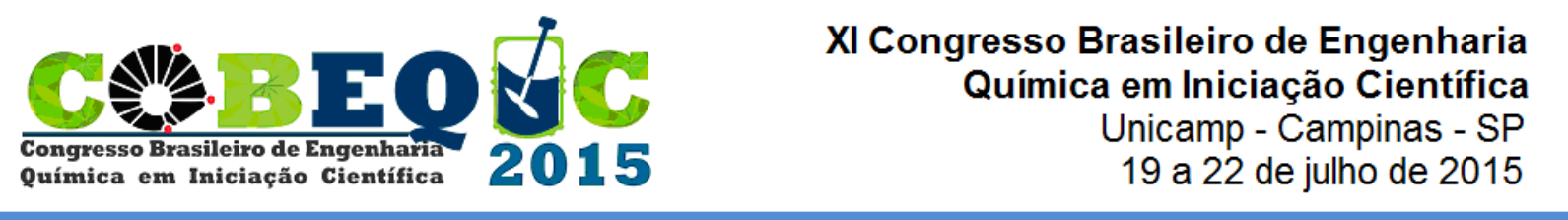

Figura 5 - Ferramenta para identificação de processos, em tempo real, via modelo ARMAX.

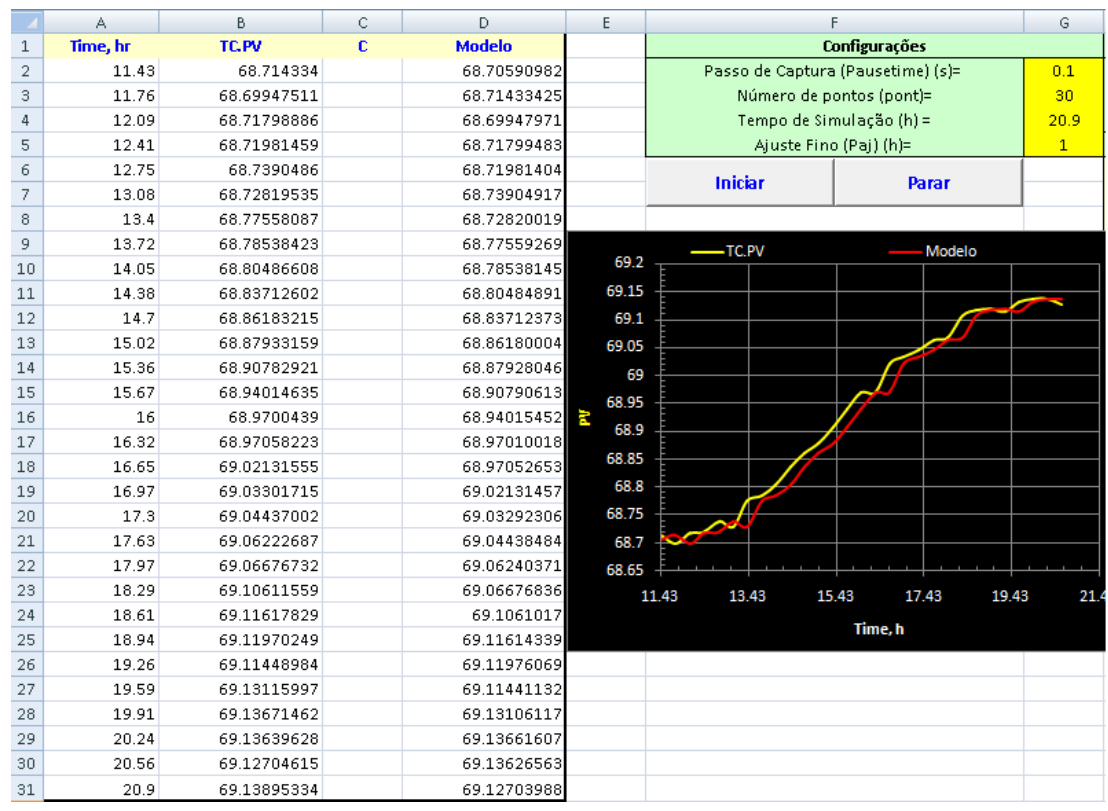

\section{CONCLUSÕES}

A ferramenta de identificação ARMAX desenvolvida apresentou uma representatividade significativa do processo, demonstrado pela adequação quanto ao acompanhamento dinâmico da variável de processo. Contudo, existem muitos outros fatores que podem influenciar no bom desempenho do controlador, estes que também devem ser estudados e seus resultados avaliados juntamente com os atuais, como, por exemplo, a auto sintonia dos parâmetros do controlador PID.

\section{REFERÊNCIAS}

ÅSTRÖM, K. J.; HÄGGLUND, T., PID Controllers: Theory, Design and Tuning, Instrument Society of America, 1995.

ÅSTRÖM, K. J.; HÄGGLUND, T., The future of PID control. Control Engineering Practice, Vol. 9, n 11, pp. 1163-1175, 2001.

CAMPOS, M. C. M. M.; TEIXEIRA, H. C. G., Controles Típicos de equipamentos e processos industriais, Editora Blucher, 2010.

OGATA, K., Engenharia de Controle Moderno, Prentice Hall do Brasil, $4^{\circ}$ ed., Rio de Janeiro, RJ, 2003.

SILVA, J., N., Determinação da Região Robusta de Estabilidade e de Desempenho inspirada nos princípios da estatística clássica, Tese (Doutorado e m Engenharia Química) Programa de Pós-Graduação em Engenharia Química, Universidade Federal de Campina Grande, Campina Grande, PB, 2013. 